\title{
Un espace « aussi vaste que fertile » : les sciences naturelles dans le rapport de Cuvier
}

Jean-Marc Drouin

\section{(2) OpenEdition Journals}

Édition électronique

URL : https://journals.openedition.org/ahrf/138

DOI : $10.4000 /$ ahrf.138

ISSN : 1952-403X

Éditeur :

Armand Colin, Société des études robespierristes

Édition imprimée

Date de publication : 1 juin 2000

Pagination : 21-31

ISSN : 0003-4436

Référence électronique

Jean-Marc Drouin, «Un espace « aussi vaste que fertile » : les sciences naturelles dans le rapport de Cuvier », Annales historiques de la Révolution française [En ligne], 320 | avril-juin 2000, mis en ligne le 23 janvier 2006, consulté le 24 avril 2022. URL : http://journals.openedition.org/ahrf/138 ; DOI : https:// doi.org/10.4000/ahrf.138

Ce document a été généré automatiquement le 24 avril 2022.

Tous droits réservés 


\title{
Un espace « aussi vaste que fertile $»$ : les sciences naturelles dans le rapport de Cuvier
}

\author{
Jean-Marc Drouin
}

«Sire. Dans l'honorable tâche que votre Majesté impériale a prescrite à l'Institut, de vous présenter un tableau général des progrès des connaissances humaines pendant les vingt dernières années, il n'est point de partie plus étendue, et par conséquent, il n'en est point de plus délicate, que celle qui embrasse les sciences purement physiques ou naturelles. ${ }^{1}$

C'est par ces mots que commence le rapport présenté par Cuvier à Napoléon en 1808 et imprimé deux ans plus tard. D'emblée le ton est donné le décor est planté, les personnages sont en place : le secrétaire perpétuel de la classe des sciences physiques s'adresse à l'Empereur et il s'apprête à traiter un champ immense du savoir. Pourtant le texte est bien plus qu'un discours de circonstance comme l'atteste sa réédition, du vivant même de son auteur mais après la chute de l'Empire ${ }^{2}$. La personnalité scientifique de Cuvier est pour beaucoup dans l'intérêt que suscite le texte ${ }^{3}$. Mais l'intérêt du rapport lui-même a été souligné, dans les dernières décennies, par plusieurs auteurs. En 1982, Jean-Pierre Gasc lui a consacré une communication éclairante et substantielle, dans un symposium paléontologique consacré à Georges Cuvier ${ }^{4}$. En 1989, dans la série des Rapports à l'Empereur, une édition présentée et annotée par Michèle Goupil, Goulven Laurent et Denis Woronoff, sous la direction d'Yves Laissus, a mis le texte à la disposition de chacun, tout en apportant informations et analyses sur le texte. En s'appuyant sur tous ces travaux, la présente contribution vise à dégager de la lecture de ce texte quelques traits saillants de la pensée de Cuvier sur la définition du domaine des sciences naturelles, ensuite sur les révolutions dont il a été le théâtre dans les deux décennies précédant le rapport, enfin de l'image de la science, de ses applications et de son éventuel caractère national. 
Le domaine des sciences naturelles

2 Cuvier, on l'a vu, se plaît à insister sur l'immensité du domaine qu'il doit couvrir. Il inclut en effet la chimie, avec l'étude de la chaleur, celle de la lumière et de l'électricité - ou du moins de leur action chimique - aussi bien que l'histoire naturelle. Celle-ci est elle-même entendue de manière extensive puisqu'elle comprend la météorologie, l'hydrologie, la minéralogie, la géologie, l'anatomie, la physiologie, aussi bien que la botanique et la zoologie.

3 Pour définir ce domaine "aussi vaste que fertile " qui s'étend entre les "sciences mathématiques» et les "sciences morales» il précise que les sciences naturelles: «commencent où les phénomènes ne sont plus susceptibles d'être mesurés avec précision, ni les résultats d'être calculés avec exactitude» et qu'elles «finissent, lorsqu'il n'y a plus à considérer que les opérations de l'esprit et leur influence sur la volonté $»^{5}$. On remarque la dissymétrie de cette délimitation: l'un des domaines limitrophes, celui des sciences mathématiques est défini par sa méthode, l'autre, celui des sciences morales, par son objet. De fait, Cuvier s'attarde peu sur ces dernières et semble les renvoyer implicitement à la poésie : «la continuelle et incompréhensible mobilité du cœur » met en défaut règles et prévisions et seul le génie semble pouvoir la fixer. Il s'attache à comparer les sciences naturelles aux sciences mathématiques. Celles-ci «ont l'avantage d'une certitude presque indépendante de l'observation », en revanche, leurs bornes sont celles du calcul de sorte que si le premier rang leur revient pour ce qui est de la certitude des résultats, il appartient aux sciences naturelles pour ce qui est de l'étendue du domaine ${ }^{6}$.

4 L'expression même de "sciences naturelles" mérite de retenir l'attention. Cuvier pourrait employer l'expression de «sciences physiques", étymologiquement équivalente et qui désigne la classe de l'Institut à laquelle il appartient et au nom de laquelle il parle. Il l'emploie quelquefois, lorsqu'il écrit, par exemple, que les «seuls principes généraux qui paroissent dominer dans les sciences physiques sont aussi ceux qui les rendent rebelles au calcul $»^{7}$. Dans les premières lignes, déjà citées, il parle de "sciences purement physiques ou naturelles". Le plus souvent cependant, il utilise simplement la formule "sciences naturelles». Or si celle-ci nous est familière, ne serait-ce que par son usage scolaire, elle ne va pas de soi au début du xixe siècle. Comme l'a rappelé récemment Pietro Corsi à propos du Dictionnaire des sciences naturelles - qui est dirigé par Frédéric Cuvier, le frère de Georges Cuvier, et dont la publication commence en 1804-1805 puis reprend en 1816 - parler de «sciences naturelles » marque une volonté de se démarquer de l'histoire naturelle spéculative et narrative des héritiers de Buffon ${ }^{8}$. Le sens de cette expression a mis longtemps à se stabiliser. En 1819, dans la préface de la seconde édition de la Théorie élémentaire de la botanique, Augustin-Pyramus de Candolle, parle de sciences "matérielles ou naturelles » à propos de l'astronomie, la minéralogie, la botanique et la zoologie, qui étudient les « corps de la nature » tandis que la physique, la chimie, la physiologie et, la psychologie se consacrent à l'étude des forces qui mettent ces corps en mouvement ${ }^{9}$.

5 En fait les sciences naturelles telles que les entend Cuvier dans son rapport incluent aussi bien l'étude des corps que celles des forces, dès lors que le jeu de celles-ci est assez compliqué pour que l'on ne puisse plus espérer parvenir à une explication mécanique opératoire, tout en sachant que les phénomènes étudiés n'échappent pas pour autant à ces forces : 
«Une fois sortis des phénomènes du choc, nous n'avons plus d'idée nette des rapports de cause et d'effet. Tout se réduit à recueillir des faits particuliers, et à chercher des propositions générales qui embrassent le plus grand nombre possible. ${ }^{10}$

6 Pour justifier sa position, Cuvier compare les effets de l'attraction d'un côté sur les astres et de l'autre sur les molécules des corps terrestres. Dans le premier cas la taille des corps célestes est si petite par rapport aux distances qui les séparent qu'on peut faire comme si toute leur masse était concentrée en un point. Dans le second, au contraire, les particules de matière sont si rapprochées que leur figure influe sur l'attraction et éloigne de nous une explication complète des phénomènes de la cohésion et des affinités chimiques, et $a$ fortiori des phénomènes qui affectent les corps vivants et sont sous l'influence des affinités chimiques.

7 À côté de cette comparaison explicite il en est une autre, implicite, que suggère la lecture conjointe du rapport de Cuvier et de celui de Jean-Baptiste Delambre sur les sciences mathématiques.

Delambre, tout en soulignant la puissance de l'outil que les fondateurs du calcul différentiel et intégral ont légué aux générations suivantes et en détaillant les applications que les astronomes et les physiciens en ont tirées, trahit un regret de n'avoir pas vécu ces découvertes qui " changent la face de la science » et de vivre une époque vouée à «des travaux immenses dont l'éclat ne sauroit jamais égaler la difficulté $»^{11}$.

9 Cuvier par contraste insiste sur l'accélération des progrès et les révolutions accomplies. Il commence par poser en principe que «les sciences ne sont que l'expression des rapports réels entre les êtres ", que leur marche est tracée et que tous les hommes qui s'y consacrent voient leurs efforts s'accumuler et converger. Pour illustrer sa conception de la connaissance, il compare la nature à un vaste tableau divisé en une infinité de compartiments liés entre eux, les sciences forment alors comme un second tableau, copie du premier mais dans lequel nombre de cases restent vides tandis que d'autres sont remplies d'images incorrectes. Tout en soulignant que les «sciences ne reproduisent encore "qu'un bien petit nombre des traits » de la nature, il se montre plein d'espoir sur leurs progrès : « les deux derniers siècles [écrit-il], ont plus fait pour elles que tous les précédents » et "les trente dernières années ont peut-être à elles seules égalé les deux derniers siècles $»^{12}$. Mais, pour le dire en termes actuels, dans ce progrès à vitesse croissante, coexistent un processus cumulatif et des réorganisations théoriques. Pour désigner ces dernières, Cuvier n'hésite pas à employer le mot de "révolution ", affirmant en conclusion qu'il n'est aucune des sciences naturelles, " qui n'ait acquis une multitude de faits précieux, de vues nouvelles », et que « la plupart ont éprouvé, dans leurs théories, des révolutions importantes qui les ont simplifiées, éclaircies, et leur ont fait faire des pas évidents vers la vérité. $»^{13}$

Les révolutions des sciences naturelles

10 Parmi les très nombreux travaux que recense Cuvier et dont l'abondance justifie l'adjectif « fertile » qu'il employait pour désigner le domaine des sciences naturelles, il est certaines innovations auxquelles il donne une importance particulière.

11 La première est la création de la cristallographie. Tout en saluant les tentatives de Romé de Lisle et de Bergman, Cuvier attribue tout le mérite de cette création à un seul auteur : René-Just Haüy ${ }^{14}$. Dès les premières lignes de la partie consacrée à la chimie, il souligne que la formation des cristaux est de tous les phénomènes liés à l'attraction 
moléculaire, le plus proche de la simplicité qu'exigent les mathématiques. Il résume ensuite la démarche d'Haüy retrouvant les formes secondaires d'un cristal par un accroissement réglé à partir d'un noyau caractéristique. Cuvier salue cette " heureuse réunion du calcul et de l'observation immédiate ", qui non seulement est « honorable pour l'esprit humain » mais qui, de plus, renouvelle la minéralogie ${ }^{15}$. Le « jeu des affinités chimiques » est aussi, aux yeux de Cuvier, un effet de l'attraction entre les particules élémentaires. L'innovation majeure dans ce domaine lui parait être la théorie de Berthollet. Celui-ci en démontrant qu'il n'y a pas d'affinités électives absolues mais que la tendance d'un corps à s'unir à d'autres change avec sa propre quantité et en fonction des facteurs physiques « rattache la chimie au grand système des sciences physiques » et la sort de son isolement initial ${ }^{16}$.

Quelle que soit l'importance qu'il donne à la théorie des affinités, la « révolution » que Cuvier place au premier rang de la chimie est celle de "la nouvelle théorie de la combustion $»^{17}$. Il s'inscrit ici dans une tradition historiographique dont Bernadette Bensaude-Vincent a montré comment elle trouvait son origine chez Lavoisier luimême ${ }^{18}$. Pour faire sentir l'importance de cette théorie, Cuvier se fait tour à tour vulgarisateur et historien. Il rappelle qu'une combustion peut être amenée par d'autres causes que la chaleur et ne pas être accompagnée de flamme :

«[...] et lorsqu'on la prend ainsi dans son acception la plus étendue, on peut dire qu'elle précède, qu'elle accompagne ou qu'elle constitue la plupart des opérations chimiques et des fonctions vitales; il n'en est presque aucune où quelque corps ne se trouve soit brûlé, soit débrûlé, si l'on peut employer ce terme expressif. » ${ }^{19}$

La révolution chimique, synthétisée et diffusée par le Traité élémentaire de chimie publié par Lavoisier en 1789, représente ainsi pour Cuvier un modèle d'innovation qu'il explicite au moyen d'une analogie :

«Jusqu'à lui, les phénomènes particuliers de la chimie pouvaient se comparer à une espèce de labyrinthe dont les allées profondes et tortueuses avaient presque toutes été parcourues par beaucoup d'hommes laborieux; mais leur point de réunion, leurs rapports entre elles et avec l'ensemble ne pouvaient être aperçus que par le génie qui saurait s'élever au-dessus de l'édifice et en saisirait le plan d'un œil d'aigle. $»^{20}$

15 Cuvier s'empresse d'affirmer la généralité de ce modèle de révolution scientifique : « tous ceux dont les grandes théories ont éclairé la nature » dit-il, ont procédé comme Lavoisier. C'est ce que confirme dans la seconde partie du rapport, celle consacrée à l'histoire naturelle, la méthode de classification d'Antoine-Laurent de Jussieu.

Si Cuvier assigne comme objet à l'histoire naturelle d'employer les « lois générales de la mécanique, de la physique et de la chimie à l'explication des phénomènes particuliers que manifestent les divers corps de la nature » il n'en souligne pas moins sa spécificité : tandis que les différentes branches de la chimie reposent sur l'expérimentation, l'histoire naturelle repose sur l'observation ${ }^{21}$. Cette conception l'amène à minorer le rôle de la physiologie, position qui, comme l'a bien montré Jean-Pierre Gasc, contraste avec son approche fonctionnaliste ${ }^{22}$. Or c'est précisément par un parallèle entre l'œuvre de Lavoisier et celle de Jussieu que Cuvier explicite cette distinction entre sciences d'expérimentation et sciences d'observation.

Bien que Cuvier n'emploie pas le mot dans ce cas-là, il est patent qu'il considère comme une révolution le succès de la méthode de classification par familles naturelles ${ }^{23}$. Tout en rappelant les tentatives antérieures, il attribue ce succès, au principe de subordination des caractères établi dans l'ouvrage d'Antoine-Laurent de Jussieu, le 
Genera Plantarum, publié en 1789. Cet ouvrage lui paraît marquer «dans les sciences d'observation, une époque peut-être aussi importante que la Chimie de Lavoisier dans les sciences d'expériences. $»^{24}$

18 Après avoir rappelé que les naturalistes considèrent comme "naturelle " une méthode qui groupe les êtres "d'après l'ensemble de leurs propriétés ou de leur organisation ", il résume la démarche de Jussieu. Celui-ci, partant de l'existence de quelques familles telles que les Graminées, les Ombellifères, les Légumineuses, «reconnues universellement pour naturelles ", a distingué les organes dont les caractères restent constants dans une même famille de ceux qui sont plus ou moins variables. Grâce à ce "calcul de l'importance des organes » il a pu ensuite constituer une centaine d'autres familles ${ }^{25}$.

19 Mais si Cuvier laisse la priorité aux botanistes dans l'établissement de la méthode naturelle, il souligne néanmoins que faute d'une connaissance suffisante de l'économie végétale, cette subordination des caractères repose en botanique essentiellement sur l'observation de la constance des caractères. En revanche en zoologie, l'anatomie comparée offre une base raisonnée à la classification :

«Outre son emploi physiologique, l'anatomie comparée en prend un très-grand pour la simple distinction des êtres. En effet, cette comparaison des organes a donné, pour chacun d'eux et pour toutes leurs parties, des caractères tels qu'une seule de ces parties peut faire reconnoître la classe, le genre et souvent l'espèce de l'animal dont elle vient. Cela devoit nécessairement être ainsi : car tous les organes d'un même animal forment un système unique dont toutes les parties se tiennent, agissent et réagissent les une sur les autres; et il ne peut y avoir de modifications dans l'une d'elles, qui n'en amènent dans toutes. " ${ }^{26}$

Dans ce résumé final de la partie sur les sciences - la suite du rapport étant consacrée aux applications - Cuvier, qui, dans les pages précédentes, a recensé les travaux des naturalistes, ceux de ses rivaux, Lamarck, Geoffroy Saint-Hilaire au même titre que les siens, ne résiste pas - en parlant de lui, comme dans l'ensemble du Rapport, à la troisième personne - à écrire :

«C'est sur ce principe qu'est fondée la méthode imaginée par M. Cuvier, pour reconnaître un animal par un seul os, par une seule facette d'os; méthode qui lui a donné de curieux résultats sur les animaux fossiles. " ${ }^{27}$

21 La formule de l'identification d'un animal par une partie de son squelette évoque les travaux de Cuvier sur les fossiles de mammifères trouvés dans les carrières de Montmartre, et en particulier la détermination d'une sarigue fossile. Cuvier raconte en 1804, dans les Annales du Muséum d'histoire naturelle, comment d'après la configuration des mâchoires et des dents de celles-ci, il put l'identifier et comment ensuite en dégageant la gangue de gypse il a pu prouver par la morphologie du bassin la justesse de cette identification. Toute la description de Cuvier tend à souligner la force prédictive du savoir anatomique : la connexion entre les organes donne à l'observation une valeur anticipative ${ }^{28}$.

Cet éclairage apporté par l'anatomie et la théorie de la terre lui permet de souligner que «toutes les sciences naturelles n'en forment réellement qu'une seule, dont les différentes branches ont des connexions plus ou moins directes, et s'éclaircissent mutuellement ${ }^{29}$. Ce qui revient à concevoir l'ensemble des sciences naturelles sur le mode d'une structure organique.

Le panorama des sciences naturelles se clôt sur une image naturaliste de leur unité.

Sciences et politique 
24 La dernière partie du Rapport, consacrée aux sciences d'application, c'est-à-dire à la médecine et à l'agriculture, est plus rapide, plus énumérative que la première partie sur la chimie ou que la seconde sur l'histoire naturelle ${ }^{30}$. Cuvier, en effet, ne néglige pas les innovations médicales et agricoles mais il les regarde essentiellement comme des applications des progrès scientifiques ou comme les objets d'une bonne administration. Il donne une importance particulière aux travaux de Jenner sur la vaccine ${ }^{31}$. Il revient même dans le résumé final sur cette découverte qui « a donné les moyens de soustraire l'humanité à l'un des plus funestes fléaux qui la tourmentaient. $»^{32}$

Aux yeux de Cuvier les sciences sont immédiatement utiles à la société, mais cette utilité immédiate est secondaire par rapport à une finalité plus haute :

«Conduire l'esprit humain à sa noble destination, la connaissance de la vérité; répandre des idées saines jusque dans les classes les moins élevées du peuple; soustraire les hommes à l'empire des préjugés et des passions; faire de la raison l'arbitre et le guide suprême de l'opinion publique, voilà l'objet essentiel des sciences; voilà comment elles concourent à avancer la civilisation, et ce qui doit leur mériter la protection des Gouvernemens qui veulent rendre leur puissance inébranlable, en la fondant sur le bien-être commun. " ${ }^{33}$

C'est dans cette perspective, qui s'inscrit dans le droit fil des Lumières, que doivent être comprises les mesures pratiques qu'il prend, avec beaucoup de précautions oratoires, la liberté de suggérer. Dans le cours du texte il a invité l'Empereur à ordonner la rédaction d'un nouveau Systema naturae, dans lequel seront décrites toutes les espèces vivantes ${ }^{34}$. Dans la page finale, il propose la création d'écoles supérieures d'agriculture et de technologie, souhaite une autonomie de l'enseignement des sciences naturelles par rapport à celui des mathématiques dans les lycées, espère l'entretien et l'accroissement « des jardins, des cabinets et des autres collections qui existent dans les départemens $»^{35}$.

Ce vibrant éloge de la science comme fondement du consensus politique et ces timides propositions politiques traduisent une même conviction que la France doit tout faire pour rester la patrie d'une science qui ne connaît pas de frontière. À travers éloges des savants et explications didactiques des découvertes, Cuvier se fait un point d'honneur de reconnaître les apports étrangers. Cependant le souci de la gloire nationale fait bon ménage avec l'universalisme puisqu'il entend que la France impériale se distingue par son impartialité et qu'il ne manque jamais de célébrer la gloire des savants français qui semblent toujours les mieux placés pour porter à leur perfection et faire converger les travaux de leurs confrères étrangers. La nouvelle théorie de la combustion mérite l'expression de chimie française, et la partie philosophique de la zoologie est en quelque sorte une science française ${ }^{36}$.

On serait tenté de penser en première lecture que Cuvier attribue essentiellement aux Allemands, les spéculations qui prétendent démontrer a priori les principes des sciences ${ }^{37}$. Toutefois comme le montrent les deux pages qu'il consacre à la critique explicite de cette philosophie de la nature, il s'étonne précisément du succès de cette doctrine "dans un pays renommé par sa raison et sa logique » et auprès d'hommes talentueux ${ }^{38}$.

29 En réalité, s'il y a chez Cuvier une science nationale qui ne se réduise pas à une politique nationale de la science, ou à une célébration des succès des savants français, il faut la chercher dans une certaine communauté de pensée dont il est une des figures marquantes. En 1808, les clivages qui se manifesteront dans les décennies suivantes 
sont encore peu apparents. Cuvier et Geoffroy ont pu signer ensemble en 1795 un mémoire "sur une nouvelle division des Mammifères " ${ }^{39}$. Certes, il ne faut pas surestimer la communauté des points de vue. Geoffroy ébauche en 1796, dans son mémoire sur les makis, une conception de l'unité de plan qu'on verrait mal approuvée par Cuvier ${ }^{40}$. Mais précisément Cuvier donne des idées de son collègue une formulation prudente que lui-même peut accepter puisqu'il cantonne aux vertébrés la recherche des analogies (nous dirions aujourd'hui des homologies) ${ }^{41}$. D'autre part, on sait que Lamarck enseigne dès 1800, des idées sur la transformation des espèces, qu'il publie l'année suivante et dont Cuvier évidemment ne souffle $\operatorname{mot}^{42}$. Mais ces clivages n'empêchent ni emprunts ni échanges, comme le montre l'approbation par Cuvier de la classification, proposée par Lamarck, des animaux sans vertèbres ${ }^{43}$. À cet égard, le rapport conforte le point de vue de Charles Gillispie sur l'unité profonde qui relie, audelà de leurs oppositions, les programmes de recherche de Cuvier, de Lamarck et de Geoffroy Saint-Hilaire ${ }^{44}$. Cette position serait sans doute confortée si on l'étendait à la botanique en y incluant Jussieu, Desfontaines et le jeune de Candolle ; et ce malgré les divergences et les rivalités qui opposent le premier aux deux autres ${ }^{45}$. À cet égard Cuvier ne témoigne pas seulement de la complémentarité des diverses sciences naturelles, mais tout autant de la complémentarité profonde qui réunit à l'intérieur de celles-ci les divers auteurs, leurs intérêts et leurs styles propres.

\section{NOTES}

1.Georges Cuvier, Rapports à l'Empereur sur le progrès des sciences, des lettres et des arts depuis 1789. II. Chimie et sciences de la nature, présentation et notes sous la dir. d'Yves Laissus, préface par Denis Woronoff, Paris, Belin, (1810) 1989, p. 35 (p.1 de l'édition de 1810 qui est indiquée en marge dans l'édition de 1989).

2.Sur les différentes éditions du rapport, voir Yves Laissus, dans G. Cuvier, op. cit., supra, $\mathrm{n}^{\circ} 1$, pp.299-300.

3.La littérature sur Cuvier est abondante et de qualité et il ne peut être question ici d'établir une bibliographie. On trouvera des références dans Henri Daudin, Cuvier et Lamarck. Les classes zoologiques et l'idée de série animale, Paris, Félix Alcan, 1926-1927, réimpression, Paris, E.A.C, 1983, 2 vol., 460-338p. Dorinda Outram, Georges Cuvier. Vocation, Science and Authority in Post-Revolutionary France, Manchester, Manchester University Press, 1984, X-300 p. Goulven Laurent, Paléontologie et Évolution en France. 1800-1860, de Cuvier-Lamarck à Darwin, Paris, CTHS, 1987, XIV-554 p. Martin Rudwick, Georges Cuvier, Fossil Bones and Geological Catastrophes. New translations and Interpretations of the Primary Texts, Chicago and London, University of Chicago Press, 1997, XVI-301 p. Signalons aussi la publication récente d'un texte de jeunesse de Cuvier Philippe Taquet, "Les premiers pas d'un naturaliste sur les sentiers du Wurtemberg : récit inédit d'un jeune étudiant nommé Georges Cuvier", Géodiversitas, 1998, vol. 20, n 2, pp. 285-318. 
4.Jean-Pierre Gasc, "Georges Cuvier acteur et spectateur dans l'évolution des sciences", in E. Buffetaut, J.-M. Mazin et E. Salmon (éds.), Actes du symposium paléontologique G. Cuvier, Montbéliard, 1983, pp. 209-219.

5.Georges Cuvier, op. cit., supra, n 1, p. 38 (pp. 5-6 de l'édition de 1810).

6.Ibid., p. 38 (p.6 de l'édition de 1810).

7.Ibid., p. 40 (p. 9 de l'édition de 1810).

8.Pietro Corsi, "Histoire naturelle continuité et rupture, 1795-1805", Colloque Rénovation et mutations des savoirs, 1795-1805, Paris, 15-16 octobre 1999.

9.Augustin-Pyramus de Candolle, Théorie élémentaire de la botanique, 2e éd., Paris, Déterville, 1819, p. 2. Sur de Candolle et la classification des sciences, voir Jean-Marc Drouin, "Classification des sciences et classification des plantes chez Augustin-Pyramus de Candolle", Revue de Synthèse, 1994, nos 1-2, pp. 149-165.

10.Georges Cuvier, op. cit. supra n 1, p. 38 (p. 7 de l'édition de 1810).

11.Jean-Baptiste Delambre, Rapports à l'Empereur sur le progrès des sciences, des lettres et des arts. I. Sciences mathématiques, présentation par Denis Woronoff et Jean Dhombres, Paris, Belin, (1810) 1989, 333 p., pp. 124-126, (pp. 129-130 de l'édition de 1810).

12.Georges Cuvier, op. cit., supra, nº 1, p. 38, (p. 6 de l'édition de 1810).

13.Ibid., p. 292 (p. 388 de l'édition de 1810).

14.Sur le rôle central de la cristallographie d'Haüy dans l'histoire naturelle de cette époque, cf. Pietro Corsi, "Models and Analogies for the Reform of Natural History. Features of the French De bate, 1790-1800", in G. Montalenti et Paolo Rossi (eds.), Lazarro Spallanzani e la biologia del Settecento, Firenze, Leo Olschki, 1982, pp. 381-396.

15.Georges Cuvier, op. cit., supra, n 1, pp. 45-50 (pp. 17-25 de l'édition de 1810).

16.Georges Cuvier, op. cit., supra, n 1, p. 55 (p. 31 de l'édition de 1810).

17.Ibid., p. 80 (pp. 68 et suiv. de l'édition de 1810).

18.Bernadette Bensaude-Vincent, Lavoisier, Mémoires d'une révolution, Préface de Michel Serres, Paris, Flammarion, 1993, 469 p. Il n'est pas possible ici de résumer ce livre capital pour la compréhension du concept de révolution scientifique. 19.Georges Cuvier, op. cit, supra, n 1, p. 80 (p. 68 de l'édition de 1810). 20.Ibid., p. 87 (p. 78 de l'édition de 1810).

21.Georges Cuvier, op. cit., supra, n 1, pp. 133-134 (p. 148 de l'édition de 1810).

22.Jean-Pierre Gasc, op. cit., supra, $n^{\circ} 4$, p. 217.

23.Sur la méthode naturelle et son importance dans l'histoire de la biologie, voir Peter F. Stevens, The Development of Biological Systematics : Antoine-Laurent de Jussieu, Nature and the Natural System, New-York, Columbia University Press, 1994, 616 p.

24.Georges Cuvier, op. cit., supra, n 1, p. 237, (p. 305 de l'édition de 1810). Cuvier fait référence ici au Traité élémentaire de chimie de Lavoisier, publié lui aussi en 1789. 25.Ibid., p. 237 (p. 306 de l'édition de 1810). Dans cette présentation pédagogique Cuvier laisse habilement de côté les différences qui pouvaient séparer ses conceptions de celles de Jussieu, en particulier le problème central de la discontinuité ou de la continuité, qu'a analysé Peter Stevens dans un article au titre évocateur : "AntoineLaurent de Jussieu et le système naturel : comment subdiviser une nature sans articulation?" (voir Peter F. Stevens, "Antoine-Laurent de Jussieu et le système naturel : comment subdiviser une nature sans articulation?", in Claude Blanckaert et al. (eds.), Le Muséum au premier siècle de son histoire, Paris, Muséum national d'histoire naturelle, Archives, 1997, pp. 241-262.

26.Georges Cuvier, op. cit., supra, n 1, pp. 252-253 (p. 330 de l'édition de 1810).

27.Ibid., pp. 252-253 (p. 330 de l'édition de 1810). 
28.Georges Cuvier, "Mémoire sur le squelette presque entier d'un petit quadrupède du genre des Sarigues, trouvé dans la pierre à plâtre des environs de Paris", Annales du Muséum d'histoire naturelle, t. V, an XIII (1804), pp. 277-292 (une planche dessinée par Cuvier). Réimprimé intégralement dans Recherches sur les ossemens fossiles de quadrupèdes, t. IIII, Paris, Deterville, 1812, paginé 1 à 16 . Sur ce texte, voir Claudine Cohen, 1997, "Stratégies et rhétoriques de la preuve dans les Recherches sur les ossements fossiles de quadrupèdes", in Claude Blanckaert et al. (eds.), Le Muséum au premier siècle de son histoire, Paris, Muséum national d'histoire naturelle, Archives, 1997, pp. 523-539 ; JeanMarc Drouin, "Les naturalistes et les lois de l'organisation", Colloque Rénovation et mutations des savoirs, 1795-1805, Paris, 15-16 octobre 1999.

29.Georges Cuvier, op. cit., supra, $n^{\circ} 1$, pp. 252-253 (p. 330 de l'édition de 1810).

30.Georges Cuvier, op. cit., supra, no 1, pp. 255-290 (pp. 330-386 de l'édition de 1810).

31.Ibid., pp. 267-268 (pp. 349-350 de l'édition de 1810).

32.Ibid., p. 293 (p. 389 de l'édition de 1810).

33.Ibid., pp. 292-293 (p. 387 de l'édition de 1810).

34.Ibid., pp. 234-235 (pp. 301-302 de l'édition de 1810).

35.Ibid., pp. 295 (p. 393 de l'édition de 1810).

36.Georges Cuvier, op. cit., supra, n 1 , p. 87 et p. 246 (p. 79 et p. 319 de l'édition de 1810). 37.Ibid., pp. 41-42 (pp. 11-12 de l'édition de 1810).

38.Ibid., pp. 190-191 (p. 235 de l'édition de 1810). Sur Cuvier, entre culture française et allemande, voir Roselyne Rey, "La circulation des idées scientifiques entre la France et l'Allemagne. Le cas Cuvier", in Jean Mondot, Jean-Marie Valentin, Jurgen Voss (eds.), Deutsche in Frankreich. Franzosen in Deutschland. 1715-1789..., Sigmaringen, Jan Thorbecke Verlag, 1992, pp. 197-208.

39.Voir Georges Cuvier et Étienne Geoffroy Saint-Hilaire, "Mémoire sur une nouvelle division des mammifères et sur les principes qui doivent servir de base dans ce travail", Magazin encylopédique, II, an 3 (1795), pp. 164-190. À cette époque, les deux auteurs ont co-signé plusieurs autres articles. Sur le conflit postérieur, voir Toby A. Appel, The Cuvier-Geoffroy Debate. French Biology in the Decades Before Darwin, New York et Oxford, Oxford University Press, 1987, $306 \mathrm{p}$.

40."Il semble que la nature s'est renfermée dans de certaines limites, et n'a formé tous les êtres vivants que sur un plan unique, essentiellement le même dans son principe, mais qu'elle a varié de mille manières dans toutes ses parties accessoires.", Étienne Geoffroy Saint-Hilaire, "Mémoire sur les rapports naturels du Makis Lemur L. et description d'une nouvelle espèce de mammifère", Magazin encylopédique, an 4 (1796), vol. 1, pp. 20-50.

41.Georges Cuvier, op. cit, supra, n. 1, pp. 251 (p. 337 de l'édition de 1810).

42.Jean-Baptiste Lamarck, Système des animaux sans vertèbres..., Paris, Déterville, 1801, VIII-432p. et Jean-Baptiste Lamarck, Recherches sur l'organisation des corps vivants, Paris, Maillard, 1802, VII-216 p. Réédition Paris, Fayard, 1986, 150 p. (Corpus des œuvres de philosophie en langue française).

43. Georges Cuvier, op. cit., supra, n. 1, p. 242 (p. 313 de l'édition de 1810).

44.Charles C. Gillispie "De l'histoire naturelle à la biologie : relations entre les programmes de recherche de Cuvier, Lamarck et Geoffroy Saint-Hilaire", in Claude Blanckaert et al. (éds.), Le Muséum au premier siècle de son histoire, Paris, Muséum national d'histoire naturelle, Archives, 1997, pp. 29-239.

45. À bien des égards, la conception morphologique d'A.P. de Candolle se rapproche de celle de Geoffroy, alors que son épistémologie rappelle celle de Cuvier. 


\section{RÉSUMÉS}

Pour Cuvier, entre les «sciences mathématiques » et les « sciences morales ", s'étend un espace " aussi vaste que fertile» celui des «sciences naturelles ». Elles incluent la chimie, l'étude de la chaleur, celle de la lumière et de l'électricité, aussi bien que l'histoire naturelle - elle-même entendue de manière extensive puisqu'elle comprend la météorologie, l'hydrologie, la minéralogie, la géologie, l'anatomie, la physiologie, aussi bien que la botanique et la zoologie. Dans le champ ainsi couvert, Cuvier souligne l'importance de l'œuvre cristallographique de Haüy et repère deux révolutions majeures. L'une, dans les sciences d'expérimentation est celle de la nouvelle chimie attachée au nom de Lavoisier. L'autre, dans les sciences d'observation est la méthode naturelle de classification systématisée par Jussieu en botanique et dont Cuvier luimême est un des promoteurs en zoologie. Les innovations techniques et médicales ne sont pas oubliées, mais sont considérées comme des applications des progrès scientifiques. À travers éloges des savants et explications didactiques des découvertes, Cuvier se fait un point d'honneur de reconnaître les apports étrangers. Cependant le souci de la gloire nationale fait bon ménage avec l'universalisme puisqu'il entend que la France impériale se distingue par son impartialité.

A space " as vast as it is fertile ». The natural sciences in Cuvier's report.

For Cuvier, between «mathematical sciences» and «moral sciences» lies a space «as vast as it is fertile, that of «natural sciences». These include chemistry, the study of heat, light and electricity, and also natural history. The broad sweep of the latter covers metereology, hydrology, mineralogy, geology, anatomy, physiology and likewise botany and zoology. In this wide field, Cuvier often emphasises the importance of the cristallographie work of Haüy and highlights two major revolutions. The first, in experimental science, is the new chemistry attached to the name of Lavoisier. The other, in the empirical sciences, is the natural method of classification systematized by Jussieu in botany and which Cuvier himself, among others, champions in zoology. Technical and medical innovations are not forgotten, but they are considered applications of scientific progress. In his eulogies of scientists and didactic explanations of discoveries, Cuvier makes a point of recognizing contributions from abroad. He manages, however, to reconcile his chauvinistic concerus with universalism by pointing out that imperial France is renowned for her impartiality.

\section{INDEX}

Mots-clés : sciences naturelles, rapports à l'Empereur, Cuvier, Haüy, Lavoisier

\section{AUTEUR}

JEAN-MARC DROUIN

Maître de conférences, Muséum national d'histoire naturelle. 